\title{
Attracting complex networks
}

\author{
G. Guerrero ${ }^{1}$, J.A. Langa ${ }^{2}$, A. Suárez ${ }^{2}$ \\ 1. Universidad Central del Ecuador, Ciudadela Universitaria, \\ Av. América S/N, Quito - Ecuador. \\ 2. Dpto. Ecuaciones Diferenciales y Análisis Numérico \\ Seville University, Spain. \\ e-mails: guerrerogiovanny@hotmail.com, langa@us.es, suarez@us.es
}

December 21, 2015

\begin{abstract}
Real phenomena from different areas of Life Sciences can be described by complex networks, whose structure is usually determining their intrinsic dynamics. On the other hand, Dynamical Systems Theory is a powerful tool for the study of evolution processes in real situations. The concept of global attractor is the central one in this theory. In the last decades there has been an intensive research in the geometrical characterization of global attractors. However, there still exists a weak connection between the asymptotic dynamics of a complex network and the structure of associated global attractors. In this paper we show that, in order to analyze the long-time behavior of the dynamics on a complex network, it is the topological and geometrical structure of the attractor the subject to take into account. In fact, given a complex network, a global attractor can be understood as the new attracting complex network which is really describing and determining the forwards dynamics of the phenomena. We illustrate our discussion with models of differential equations related to mutualistic complex networks in Economy and Ecology.
\end{abstract}

\footnotetext{
*Partially supported by FEDER and Ministerio de Economía y Competitividad grant \# MTM2011-22411, Junta de Andalucía under Proyecto de Excelencia FQM-1492 and Brazilian-European partnership in Dynamical Systems (BREUDS) from the FP7-IRSES grant of the European Union.

${ }^{\dagger}$ Partially supported by FEDER and Ministerio de Economía y Competitividad grant \# MTM2012-31304.
} 


\section{Introduction}

In any real phenomena complexity plays a crucial role, which can be characterized from the following items:

- The reality under study is composed of a set of simpler elements.

- These elements organize a network of connections, building a complex system.

- The weight of the links between nodes plays an important role.

- An finally, either the elements or the relationships between them evolves in time, i.e, the network possesses an intrinsic dynamics.

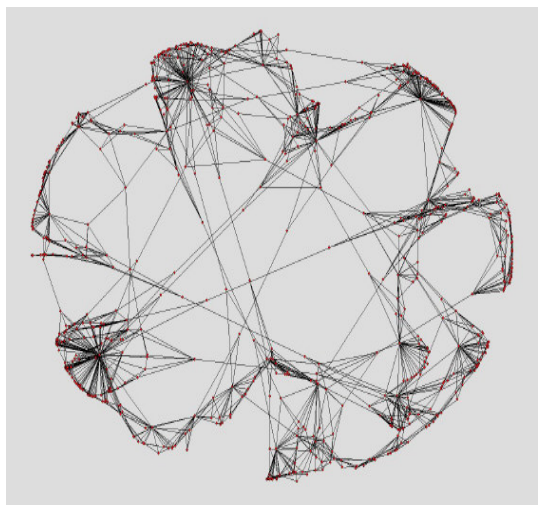

A complex network: topological approach

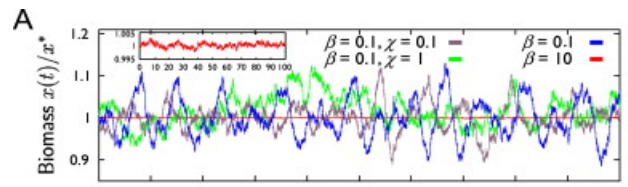

B

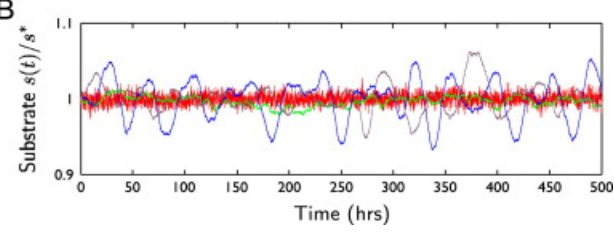

Numerical simulations: dynamical approach

Figure 1: From topology of the network to its dynamics

The description of real phenomena as complex networks has emerged as a powerful tool to understand the behavior of models in Life and Social Sciences. In particular, it is observed that there is a strong relation between the topological structure of the network (described as nodes, links and strength in connections) and the forwards dynamics of the phenomena.

On the other hand, the theory of Dynamical Systems has a long history in the Applied Mathematics. Indeed, Dynamical Systems is a very well suited methodology for modelization as we get

- From a real phenomena, a complex graph. 
- From a graph, a mathematical formulation of each expression, describing the weight of links and the dynamics of connections.

- The network is then analyzed by a system of (ordinary or partial) differential equations.

Thus, a possible strong mathematical formalism to study a Complex Network is to describe it as a Dynamical System. Indeed, the theory of dynamical systems becomes a powerful tool for the modelization of many different and important real phenomena for multiple scientific areas. In particular, the study of compact attracting invariant sets has developed a large and deep research area, providing essential information for an increasing number of models from Physics, Biology, Economics, Engineering and others. Indeed, the analysis of qualitative properties of semigroups in general phase spaces (infinite-dimensional Banach spaces or general metric spaces) has received a lot of attention throughout the last four decades (see, for instance, [5], [6], [18], [21], [23], [29], [33] or [34]). In this framework, the global attractor is a very consistent concept describing the long-time behavior of dynamical systems. A global attractor is an invariant compact set in the phase space determining all the asymptotic dynamics of the system under consideration. The study of the structure of the global attractor has received a lot of attention, going to a broad theory related to gradient systems, Morse Decomposition, Morse-Smale systems or chaotic dynamics in the attractor (see, for instance, [14, 15, 20, 29]).

Our aim in this paper is to highlight the structure of a global attractor as a complex network. Indeed, there is a natural relation between a phenomenological complex network and the (even more complex) network given by the geometrical characterization of the global attractor. 


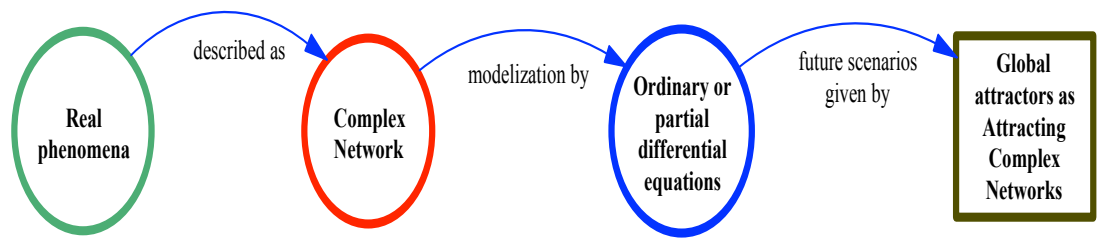

Figure 2: The dynamics of a complex network associated to a real phenomena, modelled by a system of differential equations, can be characterized by the global attractor. When available, this attracting complex network describes all the future scenarios of the phenomena.

For instance, consider the following two dimensional competitive LotkaVolterra system describing the interactions between two species which compete for the same resources

$$
\left\{\begin{array}{l}
u^{\prime}(t)=\lambda_{1} u-\beta_{1} u^{2}-\gamma_{1} u v \\
v^{\prime}(t)=\lambda_{2} v-\beta_{2} v^{2}-\gamma_{2} v u
\end{array}\right.
$$

In this model the phenomenological network would be composed just by two connected nodes. However, the long-time behavior of the system is determined by the global attractor, one of its possible structures is described in the picture below: 
Two Dimensional

Lotka-Volterra System
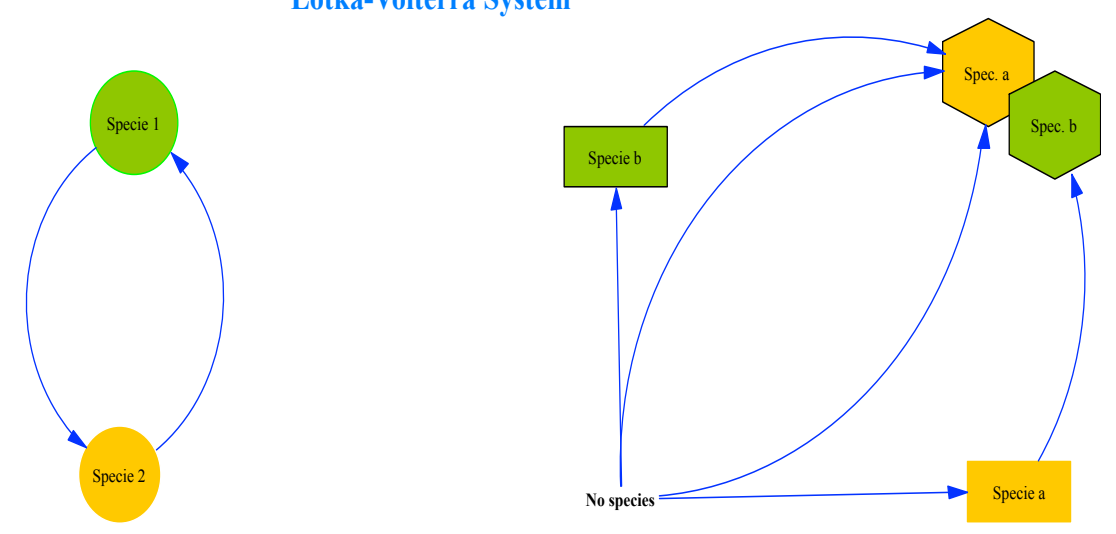

Network Connections

Network inferred by the Dynamics

Figure 3: From the real network to the dynamical complex network given by the structure of its global attractor

In this work we give mathematical evidence on the fact that is the description of these attracting complex networks what is really determining the future scenarios of the real phenomena. Thus, the global attractor, and the analysis of its structure as a dynamical complex network, emerge as a key concept to explain how the architecture of reality is transformed into an abstract attracting network determining the future behavior of the phenomena.

In Section 2 we describe the main concepts and results related to dynamical systems, the existence of the global attractor and its internal characterization. In Section 3 we describe a general model by a system of differential equations related to mutualistic complex networks associated to Ecological and Economical phenomena in which cooperation among nodes plays an essential role. We will describe the geometrical structure of the associated global attractor. To illustrate the results, in Section 4 we present a simplified three dimensional (3D) model for which all the ideas of this work can be highlighted by drawing some graphs and pictures. In a final Section we write some conclusive remarks and open questions for a further research in the near future. 


\section{Dynamical systems and attractors}

Suppose we have a system of ordinary or partial differential equations defined in a (finite or infinite-dimensional) Banach space $X$

$$
\left\{\begin{array}{l}
\frac{d u}{d t}=F(u(t)) \\
u(0)=u_{0} \in X
\end{array}\right.
$$

with $u(t)$ the unknown at time $t \geq 0$ and $F: X \rightarrow X$ a nonlinear operator on $X$. Suppose (1) has existence and uniqueness of solution $u\left(t ; u_{0}\right)$, for all $t \geq 0$.

A family $\{T(t): t \geqslant 0\}$ is called a continuous semigroup if

(a) $T(0)=I_{X}$, with $I_{X}$ being the identity in $X$,

(b) $T(t+s)=T(t) T(s)$, for all $t, s \in \mathbb{R}^{+}$and

(c) the map $\mathbb{R}^{+} \times X \ni(t, x) \mapsto T(t) x \in X$ is continuous.

$T(t)$ on $X$ describes the dynamics of each element $u \in X$. The phase space $X$ represents the framework in which the dynamics described by $T(t)$ is developed. In general, $T(t) u_{0}=u\left(t ; u_{0}\right)$ is the solution of $(1)$ at time $t$ with initial condition $u_{0}$.

\subsection{Global attractors}

First we recall the definition of a global attractor for a nonlinear semigroup

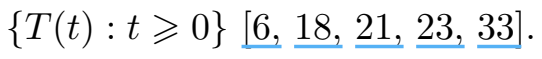

Definition 1. A set $\mathcal{A} \subseteq X$ is a global attractor for $\{T(t): t \geq 0\}$ if it is

(i) compact,

(ii) invariant under $\{T(t): t \geq 0\}$, i.e. $T(t) \mathcal{A}=\mathcal{A}$ for all $t \geq 0$, and

(iii) attracts bounded subsets of $X$ under $\{T(t): t \geq 0\}$; that is, for all $B \subset X$ bounded

$$
\lim _{t \rightarrow+\infty} \operatorname{dist}(T(t) B, \mathcal{A}) \longrightarrow 0
$$

where $\operatorname{dist}(D, A):=\sup _{d \in D} \inf _{a \in A} \operatorname{dist}(d, a)$ is the Hausdorff semidistance between two sets $D, A \subset X$. 
A global solution for a semigroup $\{T(t): t \geq 0\}$ is a continuous function $\xi: \mathbb{R} \rightarrow X$ such that $T(t) \xi(s)=\xi(t+s)$ for all $s \in \mathbb{R}$ and all $t \in \mathbb{R}^{+}$. We say that $\xi: \mathbb{R} \rightarrow X$ is a global solution through $z \in X$ if it is a global solution with $\xi(0)=z$. The global attractor can be characterized as the collection of all globally defined bounded solutions:

Lemma 2. If a semigroup $T(\cdot)$ has a global attractor $\mathcal{A}$, then

$\mathcal{A}=\{y \in X:$ there is a bounded global solution $\xi: \mathbb{R} \rightarrow X$ with $\xi(0)=y\}$.

It is well known that global attractors for semigroups are unique. For the existence, we have the following general result (see [13]).

Theorem 3. There exists a global attractor for a semigroup $T(\cdot)$ if and only if there exists a compact attracting set of bounded sets, i.e., a compact set $K \subset X$ such that $\operatorname{dist}(T(t) C, K) \rightarrow 0$ as $t \rightarrow+\infty$, for all $C \subset X$ bounded.

Definition 4. We say that $u^{*} \in X$ is an equilibrium point (or stationary solution) for the semigroup $T(t)$ if $T(t) u^{*}=u^{*}$, for all $t \geq 0$.

Definition 5. The unstable set of an invariant set $\Xi$ is defined by

$$
\begin{aligned}
& W^{\mathrm{u}}(\Xi)=\{z \in X: \text { there is a global solution } \xi: \mathbb{R} \rightarrow X \text { for } T(t) \\
& \text { satisfying } \left.\xi(0)=z \text { and such that } \lim _{t \rightarrow-\infty} \operatorname{dist}(\xi(t), \Xi)=0\right\} .
\end{aligned}
$$

\subsection{Attracting complex networks}

In this section we will describe the geometrical structure of the global attractor.

Definition 6. Let $\{T(t): t \geq 0\}$ be a semigroup on $X$. We say that an invariant set $E \subset X$ for the semigroup $\{T(t): t \geq 0\}$ is an isolated invariant set if there is an $\epsilon>0$ such that $E$ is the maximal invariant subset in the neighbourhood $\mathcal{O}_{\epsilon}(E)$.

$A$ disjoint family of isolated invariant sets is a family $\left\{E_{1}, \cdots, E_{n}\right\}$ of isolated invariant sets with the property that,

$$
\mathcal{O}_{\epsilon}\left(E_{i}\right) \cap \mathcal{O}_{\epsilon}\left(E_{j}\right)=\varnothing, \quad 1 \leq i<j \leq n,
$$

for some $\epsilon>0$. 


\subsubsection{Morse decomposition of a global attractor}

Next we introduce the notion of a Morse decomposition for the attractor $\mathcal{A}$ of a semigroup $\{T(t): t \geq 0\}$ (see [15], [25] or [29]). We start with the notion of an attractor-repeller pair.

Definition 7. Let $\{T(t): t \geq 0\}$ be a semigroup with a global attractor $\mathcal{A}$. We say that a non-empty subset $A$ of $\mathcal{A}$ is a local attractor if there is an $\epsilon>0$ such that $\omega\left(\mathcal{O}_{\epsilon}(A)\right)=A$, where $\omega(B)$ is the $\omega$-limit set of $B$, defined as

$$
\omega(B)=\left\{x \in X: S\left(t_{n}\right) x_{n} \rightarrow x, \text { for some } x_{n} \in B, t_{n} \rightarrow \infty\right\} .
$$

The repeller $A^{*}$ associated with a local attractor $A$ is the set defined by

$$
A^{*}:=\{x \in \mathcal{A}: \omega(x) \cap A=\varnothing\} .
$$

The pair $\left(A, A^{*}\right)$ is called an attractor-repeller pair for $\{T(t): t \geq 0\}$.

Note that if $A$ is a local attractor, then $A^{*}$ is closed and invariant.

Definition 8. Given an increasing family $\varnothing=A_{0} \subset A_{1} \subset \cdots \subset A_{n}=\mathcal{A}$, of $n+1$ local attractors, for $j=1, \cdots, n$, define $E_{j}:=A_{j} \cap A_{j-1}^{*}$. The ordered $n$-tuple $\mathbf{E}:=\left\{E_{1}, E_{2}, \cdots, E_{n}\right\}$ is called a Morse decomposition for $\mathcal{A}$.

An equivalent definition of a Morse decomposition for the attractor $\mathcal{A}$ of a semigroup $\{T(t): t \geq 0\}$ can be found at [2].

Definition 9. Let $\{T(t): t \geq 0\}$ be a semigroup with a global attractor $\mathcal{A}$ and $\mathbf{E}=\left\{E_{1}, E_{2}, \cdots, E_{n}\right\}$ is a Morse decomposition of $\mathcal{A}$. We say that the semigroup is dynamically gradient if for a given global solution $\xi: \mathbb{R} \rightarrow \mathcal{A}$ of $\{T(t): t \geq 0\}$

i) either $\xi(t) \in E_{i}$, for all $t \in \mathbb{R}$ and some $i=1, \cdots, n$;

ii) or there exist $1 \leq i<j \leq n$ such that $E_{j} \stackrel{t \rightarrow-\infty}{\leftarrow} \xi(t) \stackrel{t \rightarrow \infty}{\rightarrow} E_{i}$.

\subsubsection{Lyapunov functions}

Definition 10. We say that a semigroup $\{T(t): t \geq 0\}$ with a global attractor $\mathcal{A}$ and a disjoint family of isolated invariant sets $\mathbf{E}=\left\{E_{1}, \cdots, E_{n}\right\}$ is $a$ gradient semigroup with respect to $\mathbf{E}$ if there exists a continuous function $V: X \rightarrow \mathbb{R}$ such that

(i) $[0, \infty) \ni t \mapsto V(T(t) x) \in \mathbb{R}$ is non-increasing for each $x \in X$; 
(ii) $V$ is constant in $E_{i}$, for each $1 \leq i \leq n$; and

(iii) $V(T(t) x)=V(x)$ for all $t \geq 0$ if and only if $x \in \bigcup_{i=1}^{n} E_{i}$.

In this case we call $V$ a Lyapunov functional related to $\mathbf{E}$.

For gradient semigroups, the structure of the global attractor can be described as follows:

Theorem 11. Let $\{T(t): t \geq 0\}$ a gradient semigroup with respect to the finite set $\mathbf{E}:=\left\{E_{1}, E_{2}, \cdots, E_{n}\right\}$. If $\{T(t): t \geq 0\}$ has a global attractor $\mathcal{A}$, then $\mathcal{A}$ can be written as the union of the unstable manifolds related to each set in $\mathbf{E}$, i.e,

$$
\mathcal{A}=\bigcup_{j=1}^{n} W^{u}\left(E_{j}\right)
$$

Remark 12. When $E_{j}$ are equilibria $u_{j}^{*}$, the attractor is described as the union of the unstable manifolds associated to them

$$
\mathcal{A}=\bigcup_{j=1}^{n} W^{u}\left(u_{j}^{*}\right)
$$

This description shows a geometrical picture of the global attractor, in which all the stationary points are ordered by connections related to its level of attraction or stability.

Observe that each node given by a partially feasible equilibrium point in the attractor represents an attracting complex network in the original one. Thus, the attractor can be understood as a new complex dynamical network describing all the possible feasible future networks. It contains all the abstract information related to future scenarios of the model.

\subsubsection{Energy levels}

Any Morse decomposition $\mathbf{E}=\left\{E_{1}, \cdots, E_{n}\right\}$ of a compact invariant set $\mathcal{A}$ leads to a partial order among the isolated invariant sets $E_{i}$; that is, we can define an order between two isolated invariant sets $E_{i}$ and $E_{j}$ if there is a chain of global solutions

$$
\left\{\xi_{\ell}, 1 \leq \ell \leq r\right\}
$$

with

$$
\lim _{t \rightarrow-\infty} \xi_{\ell}(t)=E_{\ell}
$$


and

$$
\lim _{t \rightarrow \infty} \xi_{\ell}(t)=E_{\ell+1}
$$

$1 \leq \ell \leq r-1$, with $E_{1}=E_{i}$ and $E_{r}=E_{j}$.

This implies that, given any dynamically gradient semigroup with respect to the disjoint family of isolated invariant sets $\mathbf{E}=\left\{E_{1}, \cdots, E_{n}\right\}$, there exists a partial order in E. In Aragao et al. [3] it is shown that there exists a Morse decomposition given by the so-called energy levels $\mathbf{N}=\left\{\mathcal{N}_{1}, \mathcal{N}_{2}, \cdots, \mathcal{N}_{p}\right\}, p \leq n$. Each of the levels $\mathcal{N}_{i}, 1 \leq i \leq p$ is made of a finite union of the isolated invariant sets in $\mathbf{E}$ and $\mathbf{N}$ is totally ordered by the dynamics defined in (3). The associated Lyapunov function has different values in any two different level-sets of $\mathbf{N}$ and any two elements of $\mathbf{E}$ which are contained in the same element of $\mathbf{N}$ (same energy level) are not connected.

\subsubsection{Structural stability: robustness under perturbation}

A detailed understanding of the behaviour of isolated invariant sets and their associated unstable manifolds is one of the key facts used to prove the characterization of attractors as the union of unstable manifolds. Moreover, similar properties are used to prove that a gradient system with a finite number of hyperbolic equilibria (see $[18,19]$ ) can be completely characterized by the internal dynamics between equilibria: every global solution connects two different equilibria and there are no homoclinic structures connecting equilibria (see $[11,13]$ ).

It has been proved in $\underline{[2]}$ that a semigroup $\{T(t): t \geq 0\}$ is gradient with respect to $\mathbf{E}$ if and only if it is dynamically gradient with respect to E. Indeed, we have the following result

Theorem 13. Given a disjoint family of isolated invariant sets $\mathbf{E}=\left\{E_{1}, \cdots, E_{n}\right\}$ for a semigroup $T(t)$, the following three properties are equivalent:

i) $T(\cdot)$ is dynamically gradient;

ii) there exists an associated ordered family of local attractor-repellers; and

iii) there exists a Lyapunov functional related to $\mathbf{E}$.

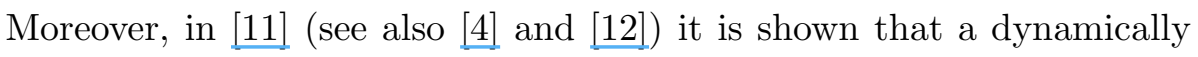
gradient system (then, a gradient one or a system with a Morse decomposition of its global attractor) is robust under small perturbation of the 
parameters and/or the linear and nonlinear operator in the equations. This

means that the structure of a gradient-like global attractor is robust under perturbation. Indeed, the results in $\underline{[11]}$ show dynamically gradient nonlinear semigroups are stable under perturbation, so that we conclude that gradient semigroups are stable under perturbation as well; that is, the existence of a continuous Lyapunov function is robust under perturbation of parameters (see $[14\rceil$ and the references therein).

\section{Mutualistic complex networks: real phenomena}

In the last decade there has been an intensive interdisciplinary research on mutualistic complex networks, mainly based on Ecology models (see, for instance, $\underline{[1}, \underline{7}, \underline{8}, \underline{9}, \underline{10}, \underline{22}, \underline{24}, \underline{28}, \underline{30}, 32])$. The results have also been applied to complex networks in Economy, in particular to the modellization of the cooperative interactions between designers and their contractors in the New York City garment industry. Indeed, this industry is characterized by a dynamic environment where resource exchanges among firms and survival depends on mutualistic connections between firms (see [26, 27]).

In general, a mutualistic network implies dozens or even hundreds of nodes building a complex net of interdependencies. In [26, 27], nodes correspond to an individual designer or contractor firm, and links between nodes indicate that a designer exchanged money for the contractors production

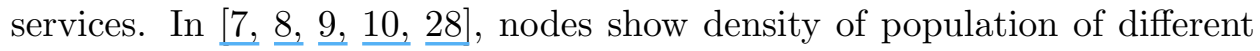
species in a particular environment. Mutualism means collaborative interaction between nodes (species) of mutual benefits for both of them. We found two different set of species (two modes network leading to bipartite graphs): plant and pollinators, or plant and seed dispersal, or firms of different business groups. 


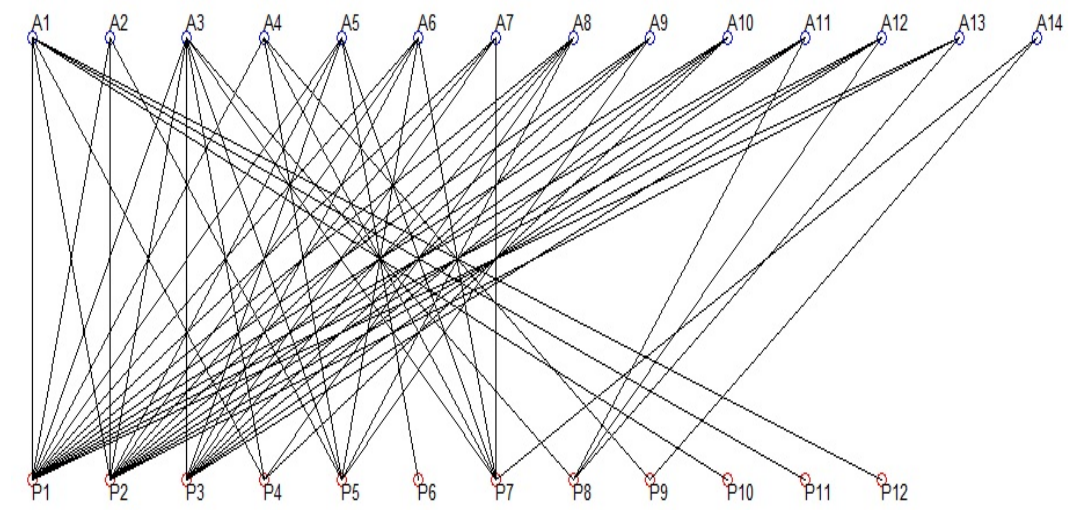

Figure 4: Mutualistic complex network: a bipartite graph; each part (A fourteen nodes- and $\mathrm{P}$-twelve nodes) is in competition among its group. Links represent cooperative interactions between the two groups in the graph.

One of the main discovers in this framework, it that, despite of its different nature, most of these mutualistic networks show a similar structure. What is more, a common architecture (similar patter formation), which in fact explains the robustness of the network $\underline{[7,} \underline{8,}, \underline{9,} \underline{10,28]}$. Robustness is defined as the strength of the net to lose its components under perturbation. This is why these complex networks in Ecology have been defined as the architecture of Biodiversity $(\underline{[9]})$.

The main characteristics of these networks are the following:

a) Distribution degree: measures the distribution of the number of connections. It is observed heterogeneity, in the sense that

i) Most of the species are specialists: they are connected to a small number of nodes. 
ii) A few species are generalists, they are connected to a huge number of nodes.

b) Nestedness: show a patron of interdependencies in which a specialist connects with subsets of the set of connections of a generalist.

c) Asymmetries: The connections between two species have different weights depending the direction of connection. Specialists of one group tend to have a strong interaction with generalists of the other group.

The consequence of this network structure is robustness. In particular, for Ecological systems:

- The nestedness configuration allows alternative routes for the persistence of the system: there exists a low number of intermediate connections to join any two nodes.

- Generalists tend to be very spread and common species, very robust to changes. This is crucial, as specialists then tend to depend of abundant species, allowing their coexistence.

- New invasive species tend also to interact with generalist, being then well integrated to the whole network.

- Asymmetries allows the network to coexist even if a specialist dies.

\subsection{The abstract model: dynamical system approach}

Bascompte and Jordano [8] (see also [24, 28]), after a description of the phenomenological properties on mutualistic complex network, introduce the following model in order to study the forwards dynamics of nodes and relations in the net.

Suppose that the $P$ nodes of a group (and the $A$ nodes of the other group) are in competition and $\mathrm{P}$-nodes and A-nodes have cooperative links.

$$
\left\{\begin{aligned}
\frac{d S_{p_{i}}}{d t} & =\alpha_{p_{i}} S_{p_{i}}-\sum_{j=1}^{P} \beta_{p_{i j}} S_{p_{i}} S_{p_{j}}+\sum_{k=1}^{A} \frac{\gamma_{p_{i k}} S_{p_{i}} S_{a_{k}}}{1+h_{P} \sum_{l=1}^{A} \gamma_{p_{i l}} S_{a_{l}}} \\
\frac{d S_{a_{i}}}{d t} & =\alpha_{a_{i}} S_{a_{i}}-\sum_{j=1}^{A} \beta_{a_{i j}} S_{a_{i}} S_{a_{j}}+\sum_{k=1}^{P} \frac{\gamma_{a_{i k}} S_{a_{i}} S_{p_{k}}}{1+h_{A} \sum_{l=1}^{P} \gamma_{a_{i l}} S_{p_{l}}} \\
S_{p_{i}}(0) & =S_{p_{i 0}}, \\
S_{a_{i}}(0) & =S_{a_{i 0}}
\end{aligned}\right.
$$


with $p_{i} \in\left\{p_{1}, \ldots, p_{P}\right\}, a_{i} \in\left\{a_{1}, \ldots, a_{A}\right\} ; \alpha_{p_{i}}$ and $\alpha_{a_{i}}$ represent the intrinsic growth rates, $\beta_{p_{i j}} \geq 0$ and $\beta_{a_{i j}} \geq 0$ competitive interactions, $\gamma_{p_{i j}} \geq 0$ and $\gamma_{a_{i j}} \geq 0$ the mutualistic interactions, $h_{A} \geq 0$ and $h_{P} \geq 0$ can be interpreted as handling times.

With this mathematical model of differential equations, which has an associated dynamical system $\{T(t): t \geq 0\}$, we are able to describe all the main structure properties of the network. Note that the original network is drawn into the abstract model, given by the positiveness or nulness of the mutualistic $\gamma_{p_{i j}}, \gamma_{a_{i j}}$ parameters, related to the associated adjacency matrix of the network.

From now on, we assume that $h_{A}=h_{P}=0$. The following results have been proved in Guerrero et al. [16]:

Theorem 14. a) Assume that $\beta=\min \left\{\beta_{p_{i j}}, \beta_{a_{i j}}\right\}<1, \gamma_{1}=\max \left\{\gamma_{p_{i j}}\right\}$, $\gamma_{2}=\max \left\{\gamma_{a_{i j}}\right\}$ and

$$
\gamma_{1} \gamma_{2}<\frac{1+\beta(P-1)}{P} \frac{1+\beta(A-1)}{A} .
$$

Then, there exists a unique positive solution of (4), for all $t>0$.

b) Assume that $\beta=\beta_{p_{i j}}=\beta_{a_{i j}}, \gamma_{1}=\gamma_{p_{i j}}, \gamma_{2}=\gamma_{a_{i j}}, \alpha=\alpha_{p_{i}}=\alpha_{a_{i}}>0$ for all $i, j$ and

$$
\gamma_{1} \gamma_{2}>\frac{1+\beta(P-1)}{P} \frac{1+\beta(A-1)}{A} .
$$

Then, any positive solution of (4) blows up in finite time.

Furthermore, in order to simplify some of the following calculations, we consider that the competition and mutualistic matrices are of mean-field type, that is $\beta_{p_{i j}}=\beta_{1}, \beta_{a_{i j}}=\beta_{2}, \gamma_{p_{i j}}=\gamma_{1}$ and $\gamma_{a_{i j}}=\gamma_{2}$.

Let $n=P+A$. Denote by $\alpha_{i}=\alpha_{p_{i}}$ for $i=1, \ldots, P$ and $\alpha_{i}=\alpha_{a_{i}}$ for $i=1, \ldots, A$. Observe that in this case, (4) can also be written as

$$
\frac{d S_{i}}{d t}=S_{i}\left(\alpha_{i}+M * S(t)\right), \quad i=1, \ldots, n,
$$

for $S(t)=\left(S_{p_{1}}(t), \ldots, S_{p_{P}}(t), S_{a_{1}}(t), \ldots, S_{a_{A}}(t)\right):=\left(S_{P}(t), S_{A}(t)\right)$, and

$$
M=\left[\begin{array}{cc}
B_{1} & \Gamma_{1} \\
\Gamma_{2} & B_{2}
\end{array}\right]_{(P+A) \times(P+A)} .
$$


where, for $k=1,2$,

$$
B_{k}=\left[\begin{array}{llll}
-1 & -\beta_{k} & \cdots & -\beta_{k} \\
-\beta_{k} & -1 & \cdots & -\beta_{k} \\
\vdots & \vdots & \ddots & \vdots \\
-\beta_{k} & -\beta_{k} & \cdots & -1
\end{array}\right]
$$

and

$$
\Gamma_{k}=\left[\begin{array}{llll}
\gamma_{k} & \gamma_{k} & \cdots & \gamma_{k} \\
\gamma_{k} & \gamma_{k} & \cdots & \gamma_{k} \\
\vdots & \vdots & \ddots & \vdots \\
\gamma_{k} & \gamma_{k} & \cdots & \gamma_{k}
\end{array}\right]
$$

As we are interested in positive solutions, we define the positive cone

$$
\mathbb{R}_{+}^{n}=\left\{u \in \mathbb{R}^{n}: u_{i} \geq 0, \text { for all } i=1, \ldots, n\right\} .
$$

Recall that, given an initial condition $\left(S_{P_{0}}, S_{A_{0}}\right)=\left\{\left(S_{p_{i 0}}, S_{a_{i 0}}\right)\{i=1, \ldots, n\}\right\}$, $T(t)\left(S_{P_{0}}, S_{A_{0}}\right)=\left(S_{P}(t), S_{A}(t)\right)$ denotes the solution of (4) at time $t$ starting in $\left(S_{P_{0}}, S_{A_{0}}\right)$ at time 0.

Theorem 15. Suppose (5), then (4) has a global attractor $\mathcal{A} \subset \mathbb{R}^{n}$ associated to $\{T(t): t \geq 0\}$.

Once we know the existence of a global attractor, we can try to describe it as an attracting complex network. In this sense, the structure of the attractor becomes the crucial complex network to take into account.

Note that the set of equilibria (stationary points) for (4) is given by solving the system of equations

$$
S_{i}\left(\alpha_{i}+M * S\right)=0, \quad i=1, \ldots, n .
$$

Denote by $\mathbf{E}:=\left\{E_{1}, E_{2}, \cdots, E_{m}\right\} \subset \mathbb{R}^{P+A}$ the set of equilibria related to (4). Observe that if $M$ is a regular matrix, at most, there is only one equilibrium with all its components strictly positive, give by the solution of the linear system $\alpha+M * S=0$, where $\alpha=\left(\alpha_{1}, \ldots, \alpha_{n}\right)$. Any other equilibria possesses, at least, one null component.

Definition 16. A matrix $M \in \mathbb{R}^{n \times n}$ is said to be Volterra-Lyapunov stable if there exists a positive diagonal matrix $D>0$ such that $D M+M^{t} D$ is negative definite, i.e., $u^{t}\left(D M+M^{t} D\right) u<0$ for all $u \in \mathbb{R}^{n}$. 
Definition 17. We say that an equilibrium $u^{*}$ is globally asymptotically stable in a region $D \subset \mathbb{R}^{n}$ if

$$
\lim _{t \rightarrow+\infty} \operatorname{dist}\left(T(t) u, u^{*}\right)=0, \text { for all } u \in D .
$$

In $\underline{[31]}$, the following result is proved:

Theorem 18. Suppose $M$ in (7) is Volterra-Lyapunov stable. Then there exists a unique globally stable equilibrium in the positive cone $\mathbb{R}_{+}^{n}$.

The following result comes from [16], and describes a Morse decomposition on global attractor for (4)

Theorem 19. Assume (5). Then

a) There exists a Morse decomposition of the global attractor on the positive cone, with $\mathbf{E}:=\left\{E_{1}, E_{2}, \cdots, E_{m}\right\}$ the set of positive equilibria.

b) The associated dynamical system is gradient with respect to $\mathbf{E}$.

c) Thus, the global attractor can be described by

$$
\mathcal{A}=\bigcup_{i=1}^{m} W^{u}\left(E_{i}\right)
$$

It is important to observe that each equilibrium is a vector in $\mathbb{R}^{P+A}$ and that its $P+A$ components correspond to the $P+A$ nodes of the phenomenological complex network. In this sense, it is remarkable that each of the stationary points is highlighting a subnet of the former complex network. Indeed, the strictly positive components of each equilibrium point out a subset of nodes and connections of the original network. In particular, the first local attractor in $\mathbf{E}$, is indeed the complex network of the phenomena showing the future biodiversity of the Ecological system, or the joint success (firms which do not dissappear) in an Economical framework.

Note that a) and (8) is not only saying that all the asymptotic behavior of the system is concentrated around $\mathcal{A}$, but it is describing the way in which the attraction takes place. In particular, it is not only showing that there exists a unique globally stable equilibrium in the positive cone $\mathbb{R}_{+}^{n}$, but how this stationary point is connected to any other, building some energy levels which organize the attraction rates. In summary, all the equilibria are ordered and oriented connected, i.e., the connections are just in one direction, determined by the forwards dynamical of the system. In this 
sense, it is the structure of the global attractor which is showing a complex network with a natural intrinsic dynamics. In fact, as each node of this attracting network is a subnet of the former network, the global attractor in this case can be understood as a network of subnetworks of the original one, which, moreover, is dynamically organized to describe all the possible future scenarios of the phenomena.

\section{A 3D model}

To illustrate our ideas, we now develop a simple model from (4) (see [17]), so that we can make some pictures in order to highlight our previous conclusions.

Thus, we consider a model consisting of three differential equations, two nodes in the first group $\left(u_{1}\right.$ and $\left.u_{2}\right)$ and just another node in the second group $\left(u_{3}\right)$ with cooperative relations with the first ones. We have

$$
\left\{\begin{array}{l}
u_{1}^{\prime}=u_{1}\left(\alpha_{1}-u_{1}-\beta u_{2}+\gamma_{1} u_{3}\right) \\
u_{2}^{\prime}=u_{2}\left(\alpha_{2}-u_{2}-\beta u_{1}+\gamma_{1} u_{3}\right) \\
u_{3}^{\prime}=u_{3}\left(\alpha_{3}-u_{3}+\gamma_{2} u_{1}+\gamma_{2} u_{2}\right) \\
\left(u_{1}(0), u_{2}(0), u_{3}(0)\right)=\left(u_{1}^{0}, u_{2}^{0}, u_{3}^{0}\right)
\end{array}\right.
$$

where $\alpha_{1}, \alpha_{2}, \alpha_{3} \in \mathbb{R}, 0<\beta<1, \gamma_{1}, \gamma_{2}>0$ and we suppose positive initial data.

Assume that

$$
\gamma_{1} \gamma_{2}<\frac{1+\beta}{2}
$$

Then, there exists a unique solution for (9), and we can define a semigroup which possesses a global attractor $\mathcal{A} \subset \mathbb{R}^{3}$. Observe that, in this case, the initial real network is just composed of three nodes, connected as show in the figure: 


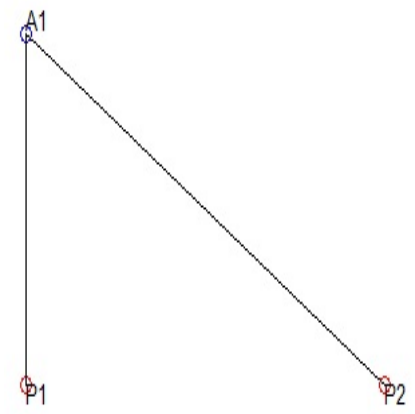

Figure 5: Phenomenological simple network in the 3D case.

For (9), it is easy to show that the eight stationary points

$$
\mathbf{E}:=\left\{E_{i j k}\right\}, \quad i, j, k=0,1,
$$

are given by

$$
\begin{gathered}
E_{000}=(0,0,0), E_{100}=\left(\alpha_{1}, 0,0\right), E_{010}=\left(0, \alpha_{2}, 0\right), E_{001}=\left(0,0, \alpha_{3}\right), \\
E_{011}=\left(0, \frac{\alpha_{2}+\gamma_{1} \alpha_{3}}{1-\gamma_{1} \gamma_{2}}, \frac{\alpha_{3}+\gamma_{2} \alpha_{2}}{1-\gamma_{1} \gamma_{2}}\right), \\
E_{101}=\left(\frac{\alpha_{1}+\gamma_{1} \alpha_{3}}{1-\gamma_{1} \gamma_{2}}, 0, \frac{\alpha_{3}+\gamma_{2} \alpha_{1}}{1-\gamma_{1} \gamma_{2}}\right), \\
E_{110}=\left(\frac{\alpha_{1}-\beta \alpha_{2}}{1-\beta^{2}}, \frac{\alpha_{2}-\beta \alpha_{1}}{1-\beta^{2}}, 0\right), \\
E_{111}=\left(\begin{array}{c}
\frac{\alpha_{1}\left(1-\gamma_{1} \gamma_{2}\right)+\alpha_{2}\left(\gamma_{1} \gamma_{2}-\beta\right)+\alpha_{3} \gamma_{1}(1-\beta)}{(1-\beta)\left(1+\beta-2 \gamma_{1} \gamma_{2}\right)} \\
\frac{\alpha_{1}\left(\gamma_{1} \gamma_{2}-\beta\right)+\alpha_{2}\left(1-\gamma_{1} \gamma_{2}\right)+\alpha_{3} \gamma_{1}(1-\beta)}{(1-\beta)\left(1+\beta-2 \gamma_{1} \gamma_{2}\right)} \\
\frac{\left(\alpha_{1}+\alpha_{2}\right) \gamma_{2}+\alpha_{3}(1+\beta)}{1+\beta-2 \gamma_{1} \gamma_{2}}
\end{array}\right)^{t} .
\end{gathered}
$$




\subsection{Stability of equilibria}

To analyze the stability of these points, we calculate the eigenvalues of the Jacobian matrix at a stationary point $\left(u_{1}, u_{2}, u_{3}\right)$ given by

$$
J\left(u_{1}, u_{2}, u_{3}\right)=\left(\begin{array}{ccc}
\alpha_{1}-2 u_{1}-\beta u_{2}+\gamma_{1} u_{3} & -\beta u_{1} & \gamma_{1} u_{1} \\
-\beta u_{2} & \alpha_{2}-2 u_{2}-\beta u_{1}+\gamma_{1} u_{3} & \gamma_{1} u_{2} \\
\gamma_{2} u_{3} & \gamma_{2} u_{3} & \alpha_{3}-2 u_{3}+\gamma_{2}\left(u_{1}+u_{2}\right)
\end{array}\right) .
$$

We have the following result (see [17]):

Theorem 20. a) Assume that the three components of $E_{111}$ are strictly positive, then $E_{111}$ is locally stable.

b) When the components of $E_{111}$ are strictly positive then the semi-trivial stationary points $E_{011}, E_{101}$ and $E_{110}$ are unstable.

c) Assume that $E_{111}$ exists. Then, it is globally asymptotically stable in the interior of $\mathbb{R}_{+}^{3}$. As a consequence, system (9) is permanent, i.e., asymptotically there exists coexistence of the three nodes.

d) The global attractor $\mathcal{A} \subset \mathbb{R}^{3}$ is given by

$$
\mathcal{A}=\bigcup_{i, j, k=0}^{1} W^{u}\left(E_{i j k}\right)
$$

This simplified model allows us to describe in detail the dependence of the associated attracting networks on parameters, showing that, in particular, to a fix phenomenological network of three species (Figure 5), corresponds a bigger and more complex set of possible future configurations given by different architectures of the global attractor, described from the equilibria and his oriented connections, as show in the Figure 6. 

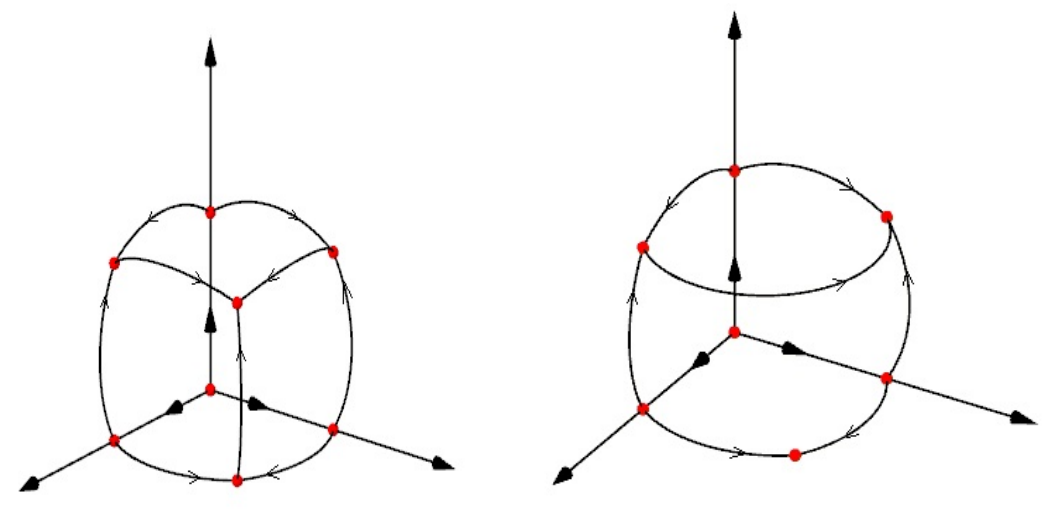

Figure 6: Two possible attracting complex networks in the 3D case. Left network shows the case in which $E_{111}$ exists and it is asymptotically globally stable; in this case $E_{111}$ is the stationary point with the lower energy level. In the right picture $E_{111}$ is not present, and the global configuration of the attracting network changes, now with $E_{011}$ as the asymptotically globally stable equilibrium. Other networks could be also reached, depending the values of the parameters in (9). Observe that all this network of possible attracting networks correspond to the same phenomenological characterization given in Figure 5 .

Item d) of Theorem 20 shows that the global attractor is gradient-like. In fact, as the associated semigroup for (9) is dynamically gradient, the stationary points in the global attractor are the Morse sets of an associated Morse decomposition of it. In particular, we can order the equilibria by energy levels (given by the associated Lyapunov functional), which describes the hierarchy on how the long-time dynamics develops with respect to positive solutions (see Figure 7). 
Level 1

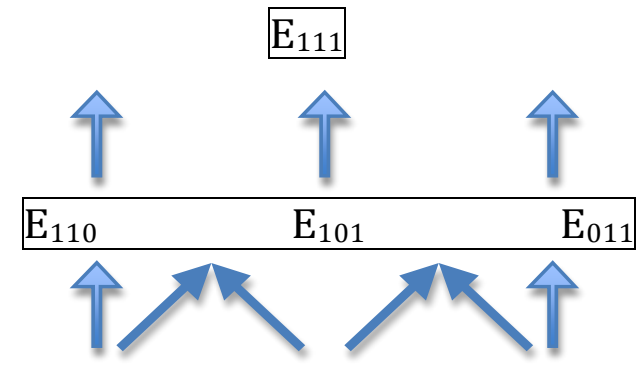

Level 2

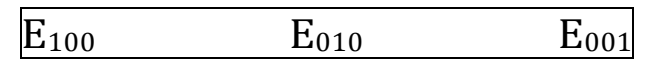

Level 4

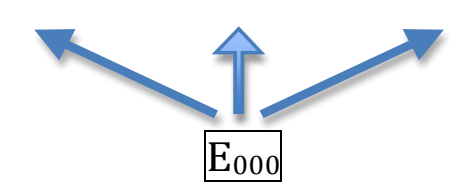

Figure 7: Organization of stationary points in the global attractor by Energy Levels. Upper level shows the minimum energy (given by the Lyapunov function), attracting every strictly positive solution. Blue arrows shows the direction of the forwards dynamics. The second level is achieved if $E_{111}$ does not exists and any of the $E_{110}, E_{101}$ or $E_{011}$ exists. The third level is only reached if any of the equilibria in upper levels are not present. $E_{000}=(0,0,0)$ is asymptotically globally stable only if any of the stationary points in an upper level exist.

\section{Conclusions}

We now enumerate some conclusions and open problems of the approach we have developed in this chapter.

a) From a real phenomena we build a complex network of nodes and connections. To model the dynamics on this complex network we build a system of ordinary or partial differential equation with an associated dynamical system.

b) A new complex network appears, described by the structure of the 
global attractor of the dynamical system, which includes dynamics and dependence on parameters.

c) New mathematically open problems appear:

i) The dependence of the structure of an attractor on parameters of the system is usually unknown.

ii) In this sense, a theory on Attractor Bifurcation on parameters would be needed.

iii) It still remains the challenging problem of dynamical complex networks, i.e., a network with time dependent parameters.

\section{References}

[1] Allesina, S., Tang, S. Stability criteria for complex ecosystems. Nature 483 (2012) 205-208.

[2] Aragão-Costa, E.R., Caraballo, T., Carvalho, A.N., \& Langa, J.A. (2011) Stability of gradient semigroups under perturbation. Nonlinearity 24, 2099-2117.

[3] Aragão-Costa, E.R., Caraballo, T., Carvalho, A.N., \& Langa, J.A. (2012) Continuity of Lyapunov functions and of energy level for a generalized gradient system. Topological Methods Nonl. Anal., 39, 57-82.

[4] Arrieta, J., Carvalho, A.N., Langa, J.A., \& Rodríguez-Bernal, A. (2012) Continuity of dynamical structures for non-autonomous evolution equations under singular perturbations. J. Dyn. Diff. Eq. 24, 427-481.

[5] Babin, A.V. \& Vishik, M. (1983) Regular attractors of semigroups and evolution equations. J. Math. Pures Appl. 62, 441-491.

[6] Babin, A.V. \& Vishik, M.I. (1992) Attractors of Evolution Equations. North Holland, Amsterdam.

[7] Bascompte, J., Jordano, P. Melián, C.J., Olesen, J.M. (2003) The nested assembly of plant-animal mutualistic networks. Proc. Natl. Acad. Sci. USA 100, 9383-9387.

[8] Bascompte, J., Jordano, P., Olesen, J.M. (2006) Asymmetric coevolutionary networks facilitate biodiversity maintenance. Science $\mathbf{3 1 2}, 431$ 433. 
[9] Bascompte, J., Jordano, P. (2007) The structure of plant-animal mutualistic networks: the architecture of biodiversity. Annu. Rev. Ecol. Evol. Syst. 38, 567-593.

[10] Bastolla, U., Fortuna, M.A., Pascual-García, A., Ferrera, A., Luque, B., Bascompte, J. (2009) The architecture of mutualistic networks minimizes competition and increases biodiversity. Nature 458, 1018-1020.

[11] Carvalho, A.N. \& Langa, J.A. (2009) An extension of the concept of gradient semigroups which is stable under perturbation. J. Diff. Eq. 246, 2646-2668.

[12] Carvalho, A.N., Langa, J.A., Robinson, J.C., \& Suárez, A. (2007) Characterization of non-autonomous attractors of a perturbed gradient system. J. Diff. Eq. 236, 570-603.

[13] Carvalho, A.N., Langa, J.A., \& Robinson, J.C. (2013) Attractors for infinite-dimensional non-autonomous dynamical systems, Applied Mathematical Series 182, Springer, New York.

[14] Carvalho, A.N., Langa, J.A., \& Robinson, J.C. (2015) Non-autonomous dynamical systems. Discrete and Cont. Dyn. Systems B 20, 703-747.

[15] Conley, C. (1978) Isolated invariant sets and the Morse index. CBMS Regional Conference Series in Mathematics Vol. 38. American Mathematical Society, Providence, R.I.

[16] Guerrero, G., Langa, J.A., Suárez, A. (2015) Architecture of attractor determines dynamics on mutualistic complex networks, preprint.

[17] Guerrero, G., Langa, J.A., Suárez, A. (2014), Biodiversity and vulnerability in a 3D mutualistic system. Discrete Contin. Dyn. Syst. 34, 4107-4126.

[18] Hale, J.K. (1988) Asymptotic Behavior of Dissipative Systems. Math. Surveys and Monographs, Amer. Math. Soc., Providence.

[19] Henry, D. (1981) Geometric theory of semilinear parabolic equations. Lecture Notes in Mathematics Vol. 840. Springer-Verlag.

[20] Hale, J.K., Magalhães, L.T., \& Oliva, W.M. (1984) An introduction to infinite-dimensional dynamical systems - geometric theory. Applied Mathematical Sciences Vol. 47. Springer-Verlag. 
[21] Ladyzhenskaya, O.A. (1991) Attractors for semigroups and evolution equations. Cambridge University Press, Cambridge, England.

[22] Okuyama, T., Holland, J.N. (2008) Network structural properties mediate the stability of mutualistic communities. Ecol. Lett. 11, 208-216.

[23] Robinson, J.C. (2001) Infinite-Dimensional Dynamical Systems. Cambridge University Press, Cambridge, England.

[24] Rohr, R.P., Saavedra, S., Bascompte, J. (2014) On the structural stability of mutualistic systems, Science 345, 1-9.

[25] Rybakowski, K.P. (1987) The homotopy index and partial differential equations. Universitext, Springer-Verlag.

[26] Saavedra, S., Reed-Tsochas, F., Uzzi, B. (2009) A simple model of bipartite cooperation for ecological and organisational networks. Nature 457, 463-466.

[27] Saavedra, S., Reed-Tsochas, F., Uzzi, B.(2009) Asymmetric disassembly and robustness in declining networks. Proc. Natl. Acad. Sci. USA 10, 16466-16471.

[28] S. Saavedra, D.B. Stouffer, B. Uzzi, and J. Bascompte (2011). Strong contributors to network persistence are the most vulnerable to extinction. Nature 478, 233-235.

[29] Sell, G.R. \& You, Y. (2002) Dynamics of evolutionary equations. Applied Mathematical Sciences Vol. 143. Springer-Verlag, New York.

[30] Suweis, S., Simini, F., Banavar, J.R., Maritan, A. (2013) Emergence of structural and dynamical properties of ecological mutualistic networks. Nature 500, 449-452.

[31] Takeuchi, Y. (1996) Global dynamical properties of Lotka-Volterra systems, World Scientific Publishing Co., Inc., River Edge, NJ.

[32] Thébault, E., Fontaine, C. (2010) Stability of ecological communities and the architecture of mutualistic and trophic networks. Science $\mathbf{3 2 9}$, 853-856.

[33] Temam, R. (1988) Infinite-Dimensional Dynamical Systems in Mechanics and Physics. Springer-Verlag, Berlin. 
[34] Vishik, M.I. (1992) Asymptotic behaviour of solutions of evolutionary equations. Cambridge University Press, Cambridge, England. 